\title{
SITUACIÓN ACTUAL DE LAS TÉCNICAS DE ENSAYO NO DESTRUCTIVO DEL HORMIGÓN
}

\author{
(CURRENT SITUATION OF THE NON-DESTRUCTIVE TECHNIQUES OF CONCRETE \\ TESTING)
}

Francisco Hostalet Alba, Dr. Ingeniero de Caminos INTEMAC - BARCELONA

Fecha de recepción: $21-$ IX - 94 Profesor Asociado del Departamento de Ingeniería de la Construcción de la ETSICCP-Barcelona ESPAÑA

\section{RESUMEN}

La investigación y normalización internacional acerca de las técnicas de ensayo del hormigón endurecido, ha experimentado un notable incremento en los últimos cincuenta y cinco años. Se describe la situación y tendencias de normalización internacional en torno a estas técnicas.

\footnotetext{
Asimismo, se analiza la situación de normalización española y se discuten algunas cuestiones relativas a las técnicas habitualmente más utilizadas en nuestro país. Finalmente, se hace una reflexión sobre la posible utilización futura de estas técnicas de ensayo, como criterios de Control de Calidad de recepción del hormigón.
}

SUMMARY

The international research and standardization of the techniques of hardened concrete testing has grown considerably in the last 55 years. This article describes the international standardization situation and tendencies concerning these techniques.

At the same time, the situation of the Spanish standardization is analyzed and some questions related to the techniques most frequently used in Spain are discussed. Finally, the article deals with the possible future use of these testing techniques as the Quality Control criteria for the reception of concrete.

\section{Introducción}

Durante los últimos cincuenta y cinco años se ha desarrollado una extensa y variada investigación sobre diferentes métodos de ensayos, destructivos (algunos autores los catalogan como semidestructivos) y no destructivos del hormigón endurecido.

Se ha pretendido con ello la puesta a punto de métodos rápidos, económicos y suficientemente versátiles (reproducibles), que permitieran conocer con el mayor grado de aproximación posible alguna propiedad física o mecánica del hormigón, a través de la cual pudiera estimarse su resistencia a compresión, asumiendo que otras muchas de sus propiedades, como son el módulo de deformación y la resistencia a tracción, a cortante y a flexotracción, están relacionadas con aquel valor.

Como consecuencia de todo ello, se desarrollaron, con diferentes grados de aceptación, técnicas como la abolladura (Williams, 1936), velocidad del impulso ultrasónico a través del hormigón (Leslie y Chesman; Jones, 1945), rebote (Schmidt, 1948) y penetración (Voellmy, 1954), siendo algo más reciente el desarrollo de la investigación relativa a las técnicas de pull-out, pull-off, break-off y método de la madurez.

A su vez han ido apareciendo y desarrollándose otras técnicas de ensayos no destructivos cuyo objetivo primordial no es la investigación de la resisten- 
cia del hormigón endurecido. Nos estamos refiriendo a los métodos magnéticos, radioactivos, ensayo de tipo químico, nucleares, eléctricos, de emisiones acústicas, técnicas de fotoelasticidad, etc., a los que se hace referencia en el apartado siguiente.

De todas las técnicas de ensayo mencionadas, las más investigadas y, en consecuencia mayormente utilizadas por conocerse mejor sus ventajas y sus limitaciones, son las correspondientes a:

- Extracción y ensayo de probetas testigo de hormigón endurecido.

- Medición de la velocidad de propagación de un impulso ultrasónico por el interior de la masa del hormigón.

- Medición del índice de rebote, mediante el esclerómetro tipo Schmidt.

Nuestro país no ha sido tampoco una excepción en la tendencia hacia la aplicación de estos métodos al reconocimiento del hormigón endurecido, pudiendo afirmarse que el resto de técnicas han sido escasamente utilizadas y que la investigación sobre su aplicación a la auscultación de hormigones se encuentra en unos estadios muy preliminares.

Los métodos de ensayo anteriormente mencionados se encuentran agrupados bajo la denominación de "Ensayos de Información", regulados en el artículo 70 de nuestra Instrucción EH-91 (11). Asimismo las normativas UNE 83.307-86 (13) y UNE 83.30886 (14), aplicables al método esclerométrico y ultrasónico, respectivamente, hacen referencia a los principales campos de aplicación de ambos métodos, procedimiento de ensayo y factores que influyen en los resultados obtenidos en los ensayos.

Para finalizar este apartado, cabe comentar algo que ya ha empezado a tener carta de identidad propia entré muchos investigadores de estos temas, y es que el estudio de la durabilidad y uniformidad del hormigón en una estructura, a través de diversas técnicas no destructivas, es tan importante como la determinación suficientemente precisa del nivel de resistencia del hormigón.

Para algunos de estos destacados investigadores, existen tres poderosas razones, que creemos vale la pena comentar, por las que la aplicación de los métodos de ensayo no destructivos a la ausculta- ción del hormigón endurecido no ha alcanzado su pleno desarrollo, e incluso ha experimentado cierto "parón" en lo que hace referencia a la investigación desarrollada tanto en Europa, como en Estados Unidos. Las razones son las siguientes:

1. Hay demasiadas técnicas de ensayos no destructivos del hormigón, todas compitiendo por su uso exclusivo, en lugar de concretar más rigurosamente el rango de variables y/o situaciones estructurales diferentes en las que cada una puede ser utilizada de forma más ventajosa desde el punto de vista técnico.

2. Hay demasiados intérpretes inexpertos de los ensayos no destructivos del hormigón, que no conocen las limitaciones físicas de los diversos ensayos y deducen conclusiones injustificadas a partir de un número de resultados muy limitado.

3. Se trata de técnicas que aún son suficientemente desconocidas (y por tanto, dejadas a los expertos) para que puedan ser introducidas como criterios de control de calidad del hormigón en las especificaciones de cada país.

\section{Métodos disponibles}

Los métodos de ensayo que son generalmente utilizados para la auscultación del hormigón endurecido, son clasificados como:

- Destructivos.

- No destructivos.

También se encuentran en la bibliografía, o en la normativa de algún país, clasificaciones en las que aparecen los denominados ensayos semidestructivos. En estos últimos casos, el término "ensayos de tipo destructivo" hace referencia a aquellos ensayos que producen una rotura de la pieza ensayada o colapso de la zona estructural investigada. Tal sería el caso, por ejemplo, del ensayo hasta rotura de elementos tipo viga o pilar en laboratorio, o la prueba de carga, hasta rotura, de un elemento o zona determinada de la estructura, aspecto este último menos habitual.

En las Tablas 1 y 2 se presenta una clasificación de los métodos de ensayo a los que se está haciendo referencia, que se han considerado como más representativos en el sentido de ofrecer una visión global de los criterios que pueden ser determinantes en la elección del método de ensayo. 
Tabla 1

\section{Clasificación de los métodos de ensayo disponibles}

\begin{tabular}{|c|c|}
\hline Información requerida & Métodos disponibles \\
\hline Comportamiento en servicio, o hasta casi-rotura de un elemento estructural. & $\begin{array}{l}\text { Prueba de carga, con medición de flechas y niveles } \\
\text { tensionales del hormigón y armaduras. }\end{array}$ \\
\hline Resistencia del hormigón & $\begin{array}{l}\text { Testigos } \\
\text { Esclerómetro } \\
\text { Ultrasonidos } \\
\text { Pull-out } \\
\text { Pull-off y Break-off } \\
\text { Resistencia a la penetración }\end{array}$ \\
\hline Propagación de la fisuración & $\begin{array}{l}\text { Ultrasonidos } \\
\text { Emisión acustica }\end{array}$ \\
\hline Coqueras internas y efectividad del proceso de compactación & $\begin{array}{l}\gamma \text { - Radiografías } \\
\text { Testigos } \\
\text { Ultrasonidos } \\
\text { Técnicas de impulso-eco }\end{array}$ \\
\hline Densidad & $\gamma$ - Radiometría \\
\hline Permeabilidad & $\begin{array}{l}\text { Ensayos de absorción } \\
\text { Ensayos de flujo (Flow Test) } \\
\text { Ensayos de capilaridad (CAPILLARY RISE TEST) }\end{array}$ \\
\hline Contenido de humedad & $\begin{array}{l}\text { Métodos nucleares } \\
\text { Resistividad eléctrica } \\
\text { Absorción de microondas }\end{array}$ \\
\hline Contenido de cemento & $\begin{array}{l}\text { Análisis químico } \\
\text { Métodos nucleares }\end{array}$ \\
\hline Propiedad de la mezcla y de sus constituyentes & $\begin{array}{l}\text { Análisis químico } \\
\text { Testigos } \\
\text { Métodos micrométricos }\end{array}$ \\
\hline Detección de armaduras & $\begin{array}{l}\text { Métodos magnéticos } \\
\text { Radiografías rayos } \mathrm{X} \\
\text { Radiografías Rayos } \gamma\end{array}$ \\
\hline Grado de deterioro del hormigón & $\begin{array}{l}\text { Análisis químico } \\
\text { Ultrasonidos } \\
\text { Métodos micrométricos }\end{array}$ \\
\hline Resistencia a la abrasión y grado de solidez & $\begin{array}{l}\text { Esclerómetro } \\
\text { Métodos físicos } \\
\text { Termografía por infrarrojos }\end{array}$ \\
\hline
\end{tabular}


Tabla 2

Principales métodos de ensayo para la estimación in situ de la resistencia del hormigón

\begin{tabular}{|c|c|c|c|}
\hline Tipo de ensayo & Método & Propiedad medida & Zona afectada por el ensayo \\
\hline \multirow{5}{*}{ SEMIDESTRUCTIVO } & Pull-Out & $\begin{array}{l}\text { Resist. a tracción, } \\
\text { comb. con esfuerzo } \\
\text { cortante }\end{array}$ & Superficial \\
\hline & Break-off & Resist. flexotracción & Superficial \\
\hline & Pull-off & Resist. a tracción & Superficial \\
\hline & Pist. Windsor & Resist. a Penetración & Superficial \\
\hline & Probetas testigo & Resist. a compresión & Zona interna \\
\hline \multirow[t]{2}{*}{ NO DESTRUCTIVO } & Esclerómetro & $\begin{array}{l}\text { Dureza superficial } \\
\text { Resiliencia }\end{array}$ & Superficial \\
\hline & Ultrasonidos & $\begin{array}{l}\text { Mod. deformación } \\
\text { dinámico }\end{array}$ & $\begin{array}{l}\text { Zona interna y } \\
\text { Zona superficial }\end{array}$ \\
\hline
\end{tabular}

\section{Elección del método de ensayo más apropiado}

Las situaciones en las que las técnicas de ensayo no destructivo del hormigón endurecido pueden ser utilizadas son muy variadas, tanto si se aplican en estructuras en construcción o de ejecución reciente, como en el caso de estructuras más antiguas.

En el momento de plantear cualquier investigación de este tipo, adquieren mucha importancia algunas consideraciones que deberían de abordarse en primer lugar, como son:

a) Motivaciones específicas. Es decir, que exista una respuesta clara a la pregunta: ¿por qué se va a realizar la investigación?

b) Objetivos perseguidos, que respondería a la pregunta: ¿para qué se hace la investigación?

La respuesta a esta pregunta, aparentemente sencilla, pero de indudable importancia, evitará confusiones posteriores, concretará el ámbito de la actuación y facilitará el establecimiento de un plan detallado de ensayos, convenientemente consensuado por las diferentes partes implicadas.

c) Métodos de ensayo disponibles y elección del método más apropiado, teniendo en cuenta las variables que intervienen en cada caso, así como los objetivos perseguidos.

d) Localización de los ensayos en las piezas a investigar y número de ensayos a realizar, actuando en una única etapa o en varias.

e) Análisis e interpretación de los resultados obtenidos en los ensayos. ¿Quién es el técnico responsable? y ¿Cómo va a procederse a esta interpretación?

De entre las consideraciones apuntadas, parece claro que las relativas a la elección del método o métodos de ensayo y el procedimiento que va a seguirse para la interpretación de los resultados, son las que van a resultar más condicionantes en el desarrollo de la investigación.

Respecto a la elección del método de ensayo merece destacarse que la normativa británica BS-1881, parte 201 (5), contiene nada menos que veinte (20) recomendaciones que pueden decidir la elección en cuestión, relacionadas con:

- Costes directos e indirectos de la realización del ensayo.

- Condiciones de la zona a investigar de la estructura. 
- Accesibilidad para la realización de un determinado ensayo.

- Efecto del posible daño producido al elemento o zona estructural que se investiga.

- Nivel de precisión requerido en la estimación del parámetro que se pretende conocer.

La asociación poco favorable de algunas de estas cuestiones, en el contexto de la investigación que se ha desarrollado sobre los métodos de ensayo no destructivo del hormigón, ha implicado que algunos de ellos, a pesar de disponer de normativa específica en algunos países (Normas BS y ASTM), hayan sido escasamente utilizados fuera del ámbito del laboratorio de ensayos. (Métodos radiográficos con rayos $\mathrm{X}$ o rayos $\gamma$, por ejemplo.)

Por otra parte, el procedimiento mediante el cual se analizan o interpretan los resultados de los ensayos es, muy probablemente, uno de los problemas más importantes a resolver cuando se pretende estimar in situ el parámetro que estamos investigando. A título de ejemplo, la interpretación de resultados de los ensayos, podría ser realizada en dos estadios diferentes, dependiendo de los resultados que se utilicen, a saber:

- Análisis de los valores obtenidos directamente de los ensayos, bien porque sea ése el plan de ensayos, bien porque se trate de una primera fase de actuación en sucesivas etapas.

- Análisis de los valores estimados para el parámetro que se investiga, obtenidos de una forma indirecta a través del establecimiento de las oportunas regresiones entre ese parámetro y la magnitud que realmente medimos en el ensayo.

En relación con este último planteamiento, parece clarividente el contexto en el que N. Petersons (18) situó la diferenciación entre dos conceptos aparentemente similares en su significado, como son los términos "fiabilidad" y "precisión", pero bien diferentes en cuanto a su adecuada interpretación. Por "fiabilidad" entiende el citado autor el grado de concordancia entre los diversos resultados obtenidos en las medidas repetidas de una determinada magnitud o parámetro, y por "precisión" el nivel de aproximación entre los valores medidos y los reales.

Señala que un método de ensayo que tenga una fiabilidad alta no tiene por qué proporcionar niveles de precisión altos en la estimación de una propiedad del hormigón, poniendo como ejemplo el método ultrasónico, sobre el que opina que tiene un grado adecuado de fiabilidad (en medición directa), pero un bajo nivel de precisión cuando se utiliza directamente para estimar la resistencia a compresión del hormigón, es decir, sin establecer la oportuna regresión con los resultados del ensayo a compresión de probetas testigo de hormigón endurecido.

\section{Normativas y recomendaciones}

En este apartado se describe en primer lugar cuál es, en líneas generales, la situación actual en cuanto a la Reglamentación Internacional que regula los campos de aplicación, utilización y análisis de las variables que influyen sobre cada una de las técnicas de ensayo semidestructivo o no destructivo del hormigón endurecido.

La referencia a la Reglamentación Internacional está basada en las normativas británicas y norteamericana, las más activas en este campo y en las recomendaciones de la RILEM.

En segundo lugar, se analiza la situación española representada por la normativa UNE y el articulado correspondiente de la vigente Instrucción EH-91 (artículo 70).

\subsection{Situación internacional. Tendencias}

Puede afirmarse, sin lugar a dudas, que dentro del contexto internacional las técnicas de ensayo del hormigón endurecido mayormente utilizadas son, tal y como ya se ha mencionado en el apartado de introducción, las relacionadas con:

— Extracción y ensayo de probetas testigo.

- Índice de rebote esclerométrico.

- Velocidad de propagación de los ultrasonidos.

Desde hace ya algún tiempo, se están manifestando sin embargo determinadas tendencias hacia la aplicación más generalizada de otros métodos, algunos de ellos relacionados con la estimación de parámetros resistentes del hormigón endurecido y otros relacionados con la detección de defectos internos del hormigón, medidas de espesores, determinaciones físico-químicas, etc.

Entre los primeros, cabe mencionar, por la importancia que están adquiriendo, los siguientes: 
Tabla 3

\section{Normativa internacional (END)}

\begin{tabular}{|c|c|c|c|}
\hline Método de ensayo & $\begin{array}{c}\text { Normativa internacional } \\
\text { (BS y ASTM) }\end{array}$ & Normativa española & Experiencia disponible \\
\hline $\begin{array}{l}\text { Esclerómetro Schmidt } \\
\text { (Suiza, 1948) }\end{array}$ & $\begin{array}{l}\text { BS 1881, Part. } 202 \\
\text { ASTM-C-805-85 } \\
\text { Recomend. RILEM NTD-3 }\end{array}$ & UNE $83307-86$ & Abundante \\
\hline \multirow{2}{*}{$\begin{array}{l}\text { Resistencia a la penetración } \\
\text { (Pistola Windsor) }\end{array}$} & \multirow{2}{*}{$\begin{array}{l}\text { BS 1881, Part. 20? } \\
\text { ASTM-C-803-02 } \\
\text { Canadá }\end{array}$} & \multirow[t]{2}{*}{ No existe } & Abundante en USA y Canadá \\
\hline & & & Escasa en Europa \\
\hline $\begin{array}{l}\text { Pull-out } \\
\text { (URSS, 1938) } \\
\text { (USA, 1944) }\end{array}$ & $\begin{array}{l}\text { BS 1881, Part. } 20^{7} \\
\text { ASTM-C-900-82 }\end{array}$ & No existe & $\begin{array}{l}\text { Abundante en algún país Europeo } \\
\text { (Dinamarca) y en USA }\end{array}$ \\
\hline $\begin{array}{l}\text { Break-off } \\
\text { (Noruega, 1976) }\end{array}$ & $\begin{array}{l}\text { BS 1881, Part. } 207 \\
\text { Noruega Suecia }\end{array}$ & No existe & Escasa \\
\hline $\begin{array}{l}\text { Método de la madurez } \\
\text { (USA, 1956) }\end{array}$ & ASTM-C-1074-8? & No existe & Escasa \\
\hline \multirow{2}{*}{$\begin{array}{l}\text { Métodos magnéticos y eléctricos } \\
\text { (1905, Francia) } \\
\text { (1919, USA) }\end{array}$} & \multirow[t]{2}{*}{$\begin{array}{l}\text { BS-1881, Part. } 204 \\
\text { ASTM-C-876-80 }\end{array}$} & \multirow[t]{2}{*}{ No existe } & Moder. para determinados ensayos \\
\hline & & & Escasa para otros \\
\hline $\begin{array}{l}\text { Métodos nucleares y radiactivos } \\
\text { (1949-1952) } \\
\text { USA }\end{array}$ & $\begin{array}{l}\text { BS-1881, Part. } 205 \\
\text { ASTM-C-1040 }\end{array}$ & No existe & $\begin{array}{l}\text { Abundante (Nucleares) } \\
\text { Moderada (Radiactivos) }\end{array}$ \\
\hline $\begin{array}{l}\text { Propagación de ondas de presión o compresión } \\
\text { (Pulso-eco, Impacto-eco, Impulso-respuesta) } \\
\text { (1960-1970) }\end{array}$ & No existe & No existe & Escasa \\
\hline $\begin{array}{l}\text { Termografía por infrarrojos } \\
\text { (Canadá, 1973) }\end{array}$ & No existe & No existe & Muy escasa \\
\hline $\begin{array}{l}\text { Métodos de frecuencia de resonancia } \\
\text { (USA, 1938) }\end{array}$ & $\begin{array}{l}\text { ASTM-C-215-85 } \\
\text { BS-1881, Part. } 209\end{array}$ & No existe & $\begin{array}{l}\text { Abundante en USA } \\
\text { Escasa en Europa }\end{array}$ \\
\hline $\begin{array}{l}\text { Propagación de ultrasonidos (1945) } \\
\text { (Canadá, Gran Bretaña) }\end{array}$ & $\begin{array}{l}\text { BS-1881, Part. } 203 \\
\text { ASTM-C-597-83 } \\
\text { RILEM NDT-1 }\end{array}$ & UNE $83308-86$ & Abundante \\
\hline Probetas testigo de hormigón endurecido & $\begin{array}{l}\text { BS 1881, Part. } 120 \\
\text { ASTM-C-42 } \\
\text { BS-1881, Part. } 201 \\
\text { ACI 437 (R-82) }\end{array}$ & $\begin{array}{l}\text { UNE-83302-84 } \\
\text { UNE-83304-84 }\end{array}$ & $\begin{array}{l}\text { Abundante } \\
\text { Escasa para testigos de pequeño diámetro }\end{array}$ \\
\hline $\begin{array}{l}\text { Métodos combinados } \\
(1966-1970) \\
\text { (Europa del Este) }\end{array}$ & $\begin{array}{l}\text { Rumania } \\
\text { Propuesta de reconocimiento } \\
\text { RILEM (París, 1983) } \\
\text { COMITE TC-43-CND }\end{array}$ & $\begin{array}{l}\text { No existe } \\
\text { (Mencionados en EH-91) }\end{array}$ & $\begin{array}{l}\text { Escasa, excepto en Rumanía y algunos } \\
\text { países de la Europa Oriental }\end{array}$ \\
\hline $\begin{array}{l}\text { Emisión acústica } \\
\text { (Alemania y Francia) (1959-1966) }\end{array}$ & No existe & No existe & Muy escasa \\
\hline Ensayos de tipo Químico del hormigón endurecido & $\begin{array}{l}\text { ASTM-C-85 } \\
\text { BS-1881, Part. } 5 \\
\text { BS-1881, Part. } 6\end{array}$ & $\begin{array}{l}\text { Varias } \\
\text { Normas UNE }\end{array}$ & $\begin{array}{l}\text { Abundante } \\
\text { (Dispersiones elevadas.) } \\
\text { (Niveles de precisión variables) }\end{array}$ \\
\hline
\end{tabular}


- Métodos combinados.

- Pull-out.

- Break-off

- Método de la madurez.

Entre los segundos deben de destacarse:

- Métodos de propagación de ondas elásticas en el interior de la masa del hormigón (pulso-eco, impacto-eco).

- Métodos magnéticos y eléctricos (medidas del potencial y velocidad de corrosión, diámetros de armaduras embebidas, etc.).

- Ensayos de tipo químico, distintos de los llamados "tradicionales".

En la Tabla 3 se presenta un resumen de la situación de normativa de los ensayos más representativos del campo del hormigón, incluyéndose en la última columna un resumen de la experiencia disponible en la aplicación de los métodos expuestos.

\subsection{Normativa española. EH-91 y normas UNE}

La aparición de las normas UNE relativas a la técnica esclerométrica y ultrasónica (UNE-83.307-86 y UNE-83.308-86), ha supuesto un paso muy importante de cara a la utilización de ambos métodos en la auscultación del hormigón endurecido, pudiendo afirmarse que, aunque mucho más escuetas y sin entrar en la fase de interpretación de resultados, están en la línea de normativa de reconocido prestigio internacional en este campo, como son las BS, ASTM y las recomendaciones RILEM.

A su vez la norma UNE-83.302-84, relativa a la extracción y preparación de probetas testigo de hormigón endurecido, ha introducido y completado de modo eficiente algunas cuestiones que no que- daban suficientemente definidas en la normativa anterior (UNE-7.240 y UNE-7.242).

Por otra parte, la vigente Instrucción IEH-91, en su artículo 70, ha mencionado (aunque sin extenderse en detalles que tal vez hubieran sido importantes) el denominado método combinado Rebote-Ultrasonidos.

Sin embargo, en las citadas normas españolas se dejan notar algunas cuestiones que a continuación se resumen y que, bien por estar presentes en estos momentos en normativas más avanzadas (BS-1.881), o bien porque la investigación recientemente desarrollada apunta a consideraciones adicionales a tener en cuenta, podrán ser objeto de futuras revisiones.

\subsubsection{Probetas testigo de hormigón endurecido}

\section{- Coeficientes de corrección por esbeltez}

Tradicionalmente, los factores de corrección por esbeltez que se aplican en el ensayo de probetas testigo de hormigón endurecido, están basados en la experimentación desarrollada en este sentido, siendo los de aplicación más frecuente los presentados por las normas americana (ASTM-C-42) y británica (BS-1.881), aunque los valores adoptados por esta última han sufrido sensibles modificaciones desde la versión antigua de 1970 hasta la más reciente de 1983 (4).

La Tabla 4, contiene los coeficientes de corrección para esbelteces de probetas testigo comprendidas entre $\lambda=1,00$ y $\lambda=2,00$, según diferentes normativas, incluida la norma UNE española.

La norma BS-1.881, parte 120 (4), que modifica los factores de corrección por esbeltez que se venían utilizando en BS-1.881 de 1970, no incluye los fac-

Tabla 4

Factores de correción por esbeltez en probetas testigo cilíndricas, según diferentes normativas

\begin{tabular}{|c|c|c|c|c|}
\hline \multirow{2}{*}{ Esbeltez } & \multicolumn{4}{|c|}{ Factores de corrección } \\
\cline { 2 - 5 } & ASTM & BS-1881 & BS-1881 & $(1983)$ \\
\hline C-42-82 & $(1970)$ & 1,00 & $\begin{array}{c}\text { UNE 833-2 } \\
(1984)\end{array}$ \\
\hline 2,00 & 1,00 & 1,00 & 0,97 & 1,00 \\
1,75 & 0,98 & 0,98 & 0,92 & 0,98 \\
1,50 & 0,96 & 0,96 & 0,87 & 0,96 \\
1,25 & 0,93 & 0,94 & 0,80 & 0,94 \\
1,00 & 0,87 & 0,92 & 0,90 \\
\hline
\end{tabular}


tores de corrección directamente. Los calcula de forma indirecta a través de la expresión:

$$
\mathrm{f}_{\mathrm{c}}=\frac{2}{1,5+1 / \lambda} \cdot \mathrm{f}_{\lambda}
$$

donde:

$\mathrm{f}_{\mathrm{C}}=$ resistencia corregida en probeta testigo cilíndrica de esbelte $z=2,00$.

$\lambda=$ esbeltez de la probeta testigo.

$\mathrm{f}_{\lambda}=$ resistencia a compresión obtenida en probeta testigo de esbeltez $\lambda$.

Por otra parte, en la Fig. 1 se presentan los resultados obtenidos en una amplia investigación desarrollada hacia principios de 1980, por J. G. Munday y R. K. Dhir (17), en relación con los factores de corrección por esbeltez a aplicar en los ensayos sobre probetas testigo.

De la citada investigación se desprende que en los coeficientes de corrección por esbeltez a aplicar, debería de tenerse en cuenta:

- El nivel de resistencia del hormigón.

- Estado de humedad del hormigón de la probeta testigo en el momento del ensayo.
- Influencia de la posible armadura embebida en la probeta testigo.

Nuestra norma UNE-83.302-84 advierte que las probetas testigo que contengan barras de armadura embebidas, pueden dar resultados diferentes de las probetas sin armadura, recomendándose que se elimine la parte de las probetas que contengan armaduras transversales al eje de la probeta, siempre que el testigo resultante tenga una esbeltez en el ensayo mayor de la unidad.

Para los casos en que no es posible obtener testigos sin armadura transversal embebida (por ejemplo, armadura en el centro del testigo), o cuando los condicionantes de esbeltez nos impidan eliminar la armadura, la norma UNE no cuantifica el efecto.

A este respecto las normas británicas BS-1.881, part. 120 (4) y la BS-6.089 (3) dan indicaciones precisas de cómo cuantificar el efecto de la presencia de la armadura transversal y su posición en la probeta testigo.

Las investigaciones más interesantes desarrolladas hasta este momento, apuntan a un aumento de la dispersión en los resultados de los ensayos a compresión si el testigo presenta, en el momento del ensayo, armadura transversal. En cualquier caso, cabe imponer al diámetro de las barras las mismas

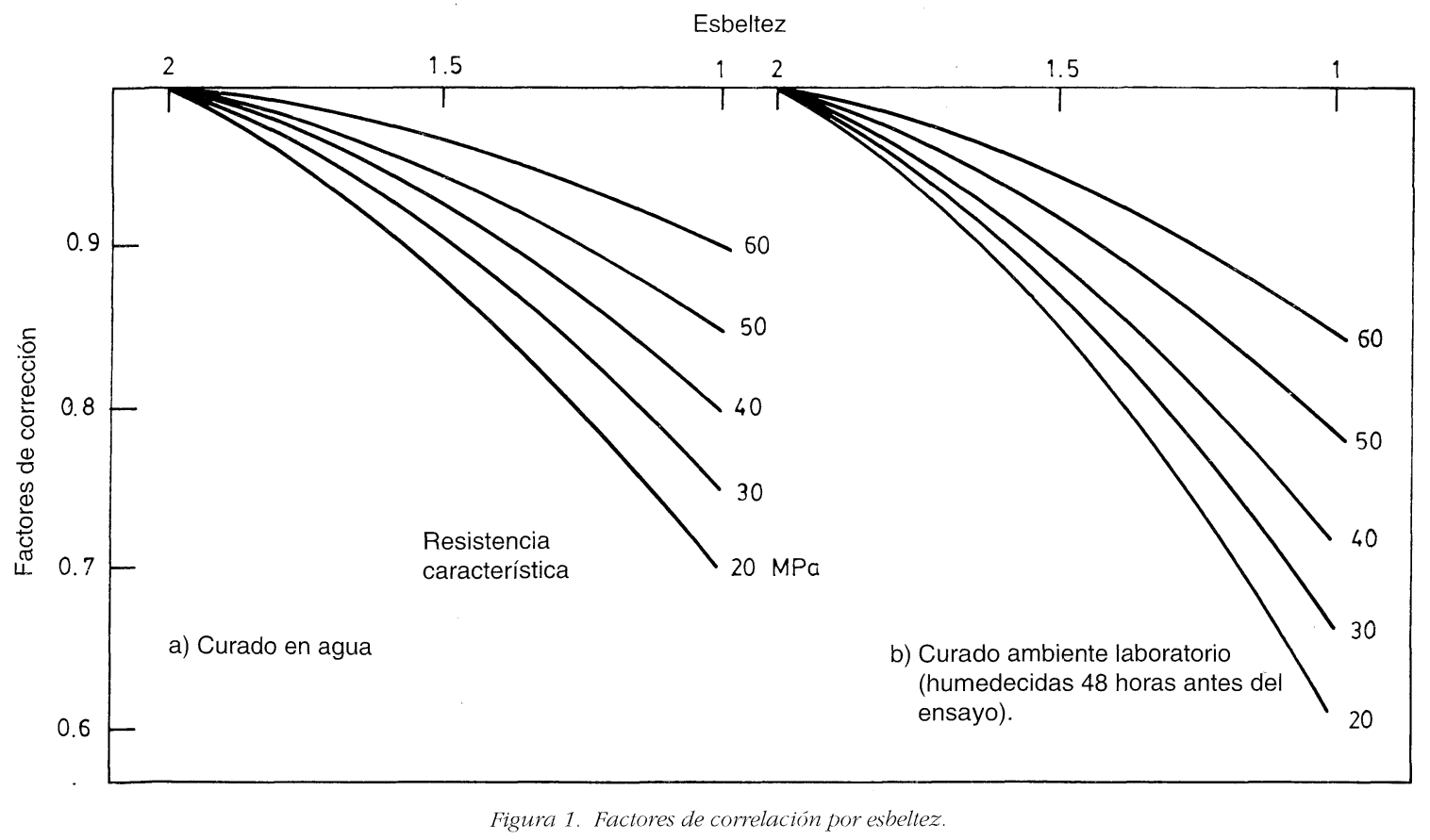



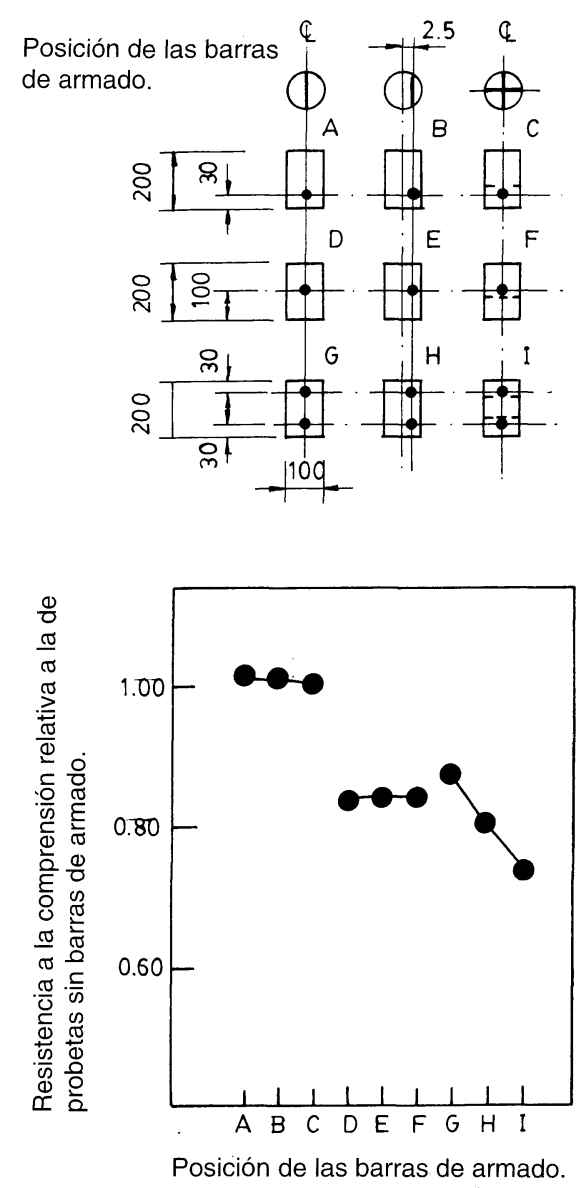

Figura 2. Relación entre la posición de las barras de armadura y la resistencia a la compresión relativa.

limitaciones, respecto al diámetro del testigo, que para el tamaño máximo del árido ( $\varnothing /$ t. max. $\geq 3)$.

En la Fig. 2 se esquematizan los resultados de una investigación japonesa sobre la influencia de esta variable, aportando datos muy interesantes al respecto. También en la referencia (9), pueden encontrarse aspectos concluyentes.

\section{- Influencia del tipo de elemento estructural y de la posición del testigo en la pieza.}

Es ésta otra de las variables importantes que pueden afectar al resultado que se obtiene en el ensayo a compresión de probetas testigo de hormigón endurecido. Probablemente, ésta y otras variables, que adquieren especial significación según los objetivos perseguidos en cada investigación, no están contempladas en nuestra normativa porque es una norma de "extracción, preparación y conservación de probetas testigo" y no una norma de interpretación de resultados que permita proceder a una estimación razonable y suficientemente precisa de la resistencia del hormigón, a través del ensayo a compresión de los testigos.
A efectos comparativos, también la normativa británica y americana contemplan las operaciones de extracción y preparación para el ensayo de las probetas testigo, pero disponen de normas complementarias de interpretación de los resultados y estimación de resistencias a compresión del hormigón investigado en obra.

Es ésta, por tanto, una asignatura pendiente de nuestra normativa que contribuiría a un tratamiento más uniforme desde el punto de vista técnico, de los resultados obtenidos en cualquier investigación de la calidad del hormigón endurecido.

\section{- Influencia del diámetro de la probeta testigo.}

En nuestra norma UNE-83.302-84, al igual que en otras normativas internåcionales, se recomienda que el diámetro de las probetas testigo sea igual o superior a $100 \mathrm{~mm}$.

Actualmente, sin embargo, ya se dispone de información, a través de las investigaciones desarrolladas al respecto, sobre la posibilidad de obtener probetas testigo de pequeño diámetro $(\varnothing \simeq 50 \mathrm{~mm}$ ), para la estimación in situ de la resistencia del hormigón endurecido.

Pueden aducirse a priori ciertas ventajas al utilizar probetas testigo de pequeño diámetro, como son:

- Pueden utilizarse máquinas o sondas de extracción de menor potencia y, por tanto, de menor coste de adquisición y/o amortización.

- Es más fácil evitar el corte de armaduras en las operaciones de extracción.

- En estructuras en servicio, el daño causado al elemento estructural, como consecuencia de la realización del taladro, es menor.

- En numerosas ocasiones es prácticamente imposible recurrir a probetas testigo de diámetros convencionales ( $\varnothing 75,100,150 \mathrm{~mm})$, bien motivado por la esbeltez de los elementos estructurales que se investigan, bien debido a la cuantía de armadura colocada en la zona del elemento estructural accesible a la inspección.

El resumen de las evidencias de que se dispone en relación con testigos de pequeño diámetro $(\varnothing \simeq 45$ a $50 \mathrm{~mm}$ ), permite afirmar que, en general, la resistencia media a compresión a través de ensayo de testigos de diferentes diámetros ( $\varnothing$ 50, 75, 100, 150 
$\mathrm{mm}$ ) no se ve muy afectada, aunque las probetas de diámetros pequeños presentan mayores dispersiones, por lo que el número de probetas testigo que deberían de obtenerse sería el triple de las que se considerarían adecuadas con diámetros convencionales, a efectos de mantener en valores razonables los niveles de precisión alcanzados en uno u otro caso.

\subsubsection{Técnica esclerométrica}

La aparición de nuestra norma UNE-83.307-86 ha supuesto, como se ha mencionado anteriormente, un paso importante en la aplicación, procedimiento de ensayo y enumeración de las variables que puedan influir sobre los resultados que se obtienen en el ensayo con el esclerómetro Schmidt.

Sin embargo, de nuevo nos encontramos con una norma que no cuantifica las diversas influencias de estas variables (algunas más determinantes que otras, dependiendo de cada investigación), ni establece ningún procedimiento para la interpretación de resultados de los ensayos, con vistas a la estimación in situ de la resistencia del hormigón, que es una de las aplicaciones más controvertidas de esta técnica y, al mismo tiempo, la más utilizada.

Por el contrario, sí que indica que la técnica no debe de sustituir a los ensayos destructivos (semidestructivos) empleados para determinar la resistencia del hormigón, sino que más bien debe utilizarse como método adicional o complementario.

También se precisa que la resistencia sólo podrá estimarse si es posible establecer para cada hormigón investigado una correlación fiable, con ensayos sobre probetas testigo o sobre probetas moldeadas realizadas durante la construcción, representativas de la zona estructural en cuestión y curadas en las mismas condiciones que el hormigón de la obra.

Con vistas al establecimiento de estas regresiones entre ensayos destructivos y no destructivos, el técnico responsable del asunto se pregunta lo siguiente:

- ¿Cúal es el mínimo número de testigos recomendable para poder obtener una correlación fiable y que proporcione unos valores adecuados de precisión en la estimación in situ de las resistencias a compresión del hormigón?

- Para un mismo hormigón ¿las correlaciones matemáticas que se obtienen, son las mismas o similares, según que se establezcan en base al ensayo de probetas testigo o al ensayo sobre probetas moldeadas curadas en ambiente de obra? ¿Los niveles de precisión pueden ser similares o comparables?

- ¿Cómo intervienen en estos procesos de calibración o estimación de resistencias a través de regresiones, variables como la edad del hormigón, su masa específica o el nivel de resistencia a compresión?

Todas estas consideraciones, que son muy habituales en nuestra práctica profesional del día a día, deben hacernos reflexionar sobre la oportunidad y sobre la imperiosa necesidad de disponer de una normativa más amplia, con criterios de aplicación e interpretación, similares a los contenidos en las recomendaciones RILEM NTD-3 (20), o en la normativa británica BS-1.881, part. 202 (6), por poner dos ejemplos de normativas suficientemente prestigiadas, basadas en la gran cantidad de investigación internacional desarrollada hasta estos momentos.

\subsubsection{Técnica ultrasónica}

Los campos de aplicación, fundamento del método, procedimiento operatorio y variables que afectan a los resultados que se obtienen en el ensayo, quedan descritos en nuestra norma UNE 83.308-86.

Respecto a este método y a su normativa española específica, cabe realizar los mismos comentarios que en la técnica esclerométrica en relación con el análisis, interpretación de resultados y establecimiento de las oportunas correlaciones entre ensayos destructivos y no destructivos, si el objetivo de la investigación es la estimación in situ de la resistencia del hormigón.

Por otra parte, sí parece importante considerar aquí la influencia del efecto de las armaduras en los valores medidos de la velocidad del pulso ultrasónico, ya que se trata de una variable muy importante en muchos casos y que nuestra normativa únicamente "advierte" de este efecto sin entrar en detalles que son fundamentales.

Conviene aclarar, a este respecto, que la normativa británica actual (BS-1.881, part. 203) ha variado por completo el rumbo, en la cuantificación del efecto de la presencia de armadura, desde su versión antigua de 1974, BS-4.408, part. 5. Las Figs. 3, 4, 5 y 6 


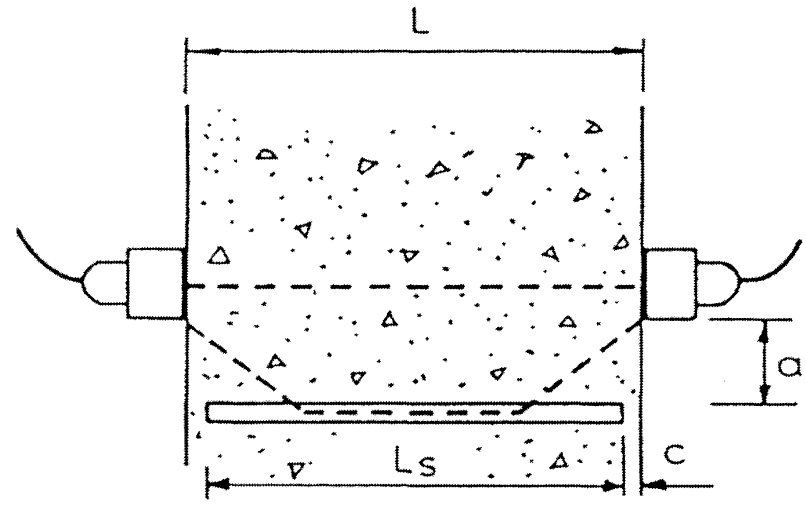

Figura 3. Barras paralelas al impulso ultrasónico.

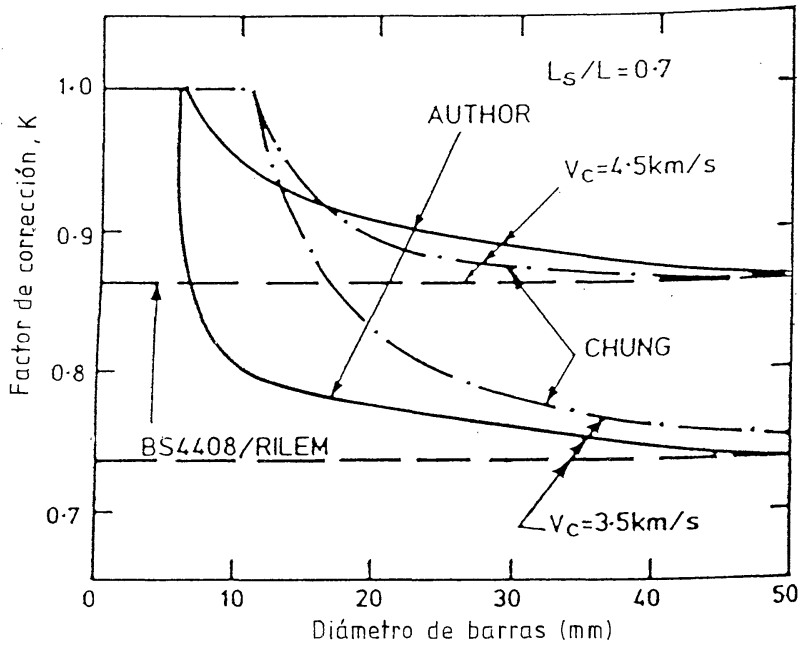

Figura 4. Comparación de los factores de corrección K para barras paralelas al impulso ultrasónico.

presentan el resumen de las investigaciones desarrolladas por J. H. Bungey (8), que también hace referencia a las realizadas en este mismo sentido por H. W. Chung (10).

Las investigaciones de J. H. Bungey, han sido tomadas por la norma BS-1.881, part. 203 (7). Por otra parte, las recomendaciones de RILEM NDT-1 (19), que datan de 1972, también contienen datos específicos sobre la posible afección de las armaduras (paralelas o perpendiculares a la trayectoria del impulso) sobre los valores de velocidad de paso de los ultrasonidos a través del hormigón endurecido.

\subsubsection{Método combinado}

En la actualidad el método combinado más utilizado es el que relaciona el índice de rebote esclerométrico, la velocidad del impulso ultrasónico y la resistencia a compresión del hormigón. Ello ha quedado confirmado a través de la encuesta que, sobre

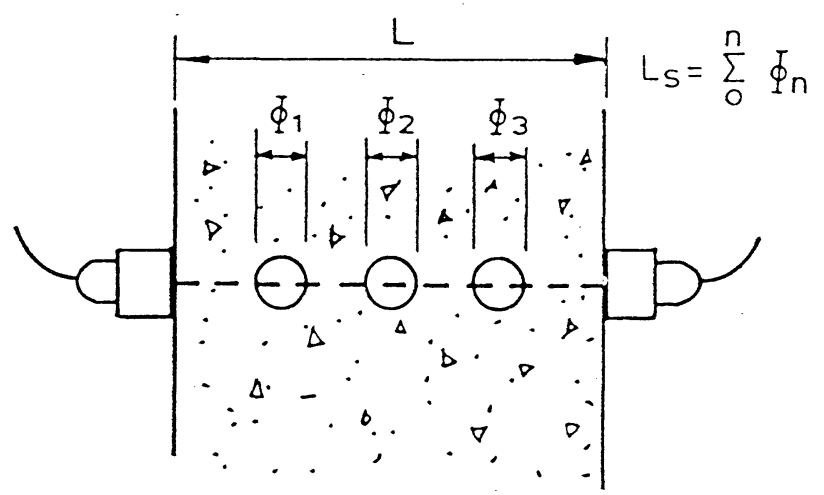

Figura 5. Barras transversales al impulso ultrasónico.

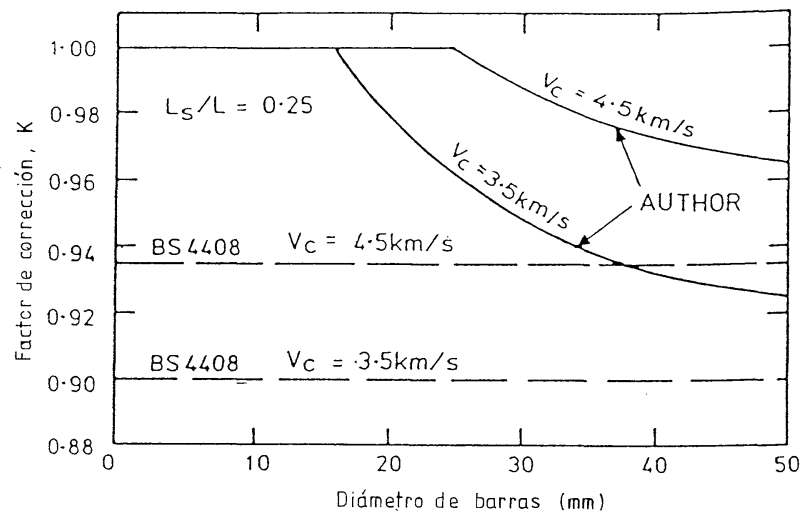

Figura 6. Comparación de factores corrección para barras situadas transversalmente al impulso.

esta combinación de ensayos, realizaron R. Jones e I. Facaoaru (15) en 1968, en el seno de RILEM.

El método ha sido ampliamente experimentado en Rumanía y en algunos países de la Europa Oriental y, más recientemente, ha sido estudiada su aplicación específica en países como Italia, Holanda y España.

Su rápida divulgación y el alto nivel de aceptación que ha obtenido en pocos años —que ha conducido a que sea incluido o mencionado en la normativa sobre ensayos no destructivos de algunos países- están basados en el potencial aumento del nivel de precisión que se obtiene en la estimación in situ de la resistencia del hormigón, frente a la utilización del método de dureza superficial o propagación de los ultrasonidos, utilizados de forma aislada.

No obstante, también este método es contemplado por algunos expertos con determinadas dosis de 
escepticismo, sobre todo cuando se utiliza para estimar in situ la resistencia de hormigones de avanzada edad, debido al efecto combinado de la posible carbonatación de la superficie del hormigón, que afecta a los valores del Índice de Rebote y a la falta de sensibilidad de los ultrasonidos para detectar cambios sustanciales de resistencia, cuando la matriz de cementación del hormigón ha finalizado su proceso de hidratación.

En los comentarios al artículo 70 de nuestra Instrucción EH-91, se indica que la fiabilidad en la estimación de la resistencia del hormigón aumenta cuando se combinan las técnicas de rebote y ultrasonidos, con la extracción y ensayo a compresión de probetas testigo (método combinado).

El término "fiabilidad" puede interpretarse en el doble sentido de que, por una parte, se combinan técnicas suficientemente conocidas e investigadas y, por otra parte, el método debe proporcionar niveles de precisión, en la estimación de resistencias a compresión del hormigón, que puedan considerarse satisfactorias, dependiendo del objetivo perseguido.

Sin embargo, en el estado actual del conocimiento, los niveles de precisión que muchos autores confieren a esta técnica del método combinado, han sido obtenidos a partir de investigaciones desarrolladas, en su mayor parte en laboratorio y, por tanto, insuficientemente contactadas en su aplicación a estructuras reales, aspecto este último, en el que nuestro país tampoco es una excepción.

\section{Utilización de los END como criterios de control de calidad}

A pesar de lo mucho que se ha investigado sobre la aplicación de algunas técnicas de ensayo semidestructivo o no destructivo a la estimación de determinados parámetros resistentes o de durabilidad del hormigón endurecido, la realidad es que estas técnicas son muy raramente utilizadas como criterios de Control de Calidad, en base a los cuales puedan establecerse juicios de aceptación o rechazo.

Es el caso de las normativas Canadiense, Australiana, Japonesa y la de Estados Unidos, que no contemplan la utilización de estas técnicas como instrumento de Control de Calidad. Las normativas Europeas en general y la Británica en particular, muy activa en este sentido, no son diferentes de las anteriores constituyendo la excepción algunos países de la Europa Oriental, entre los que merece destacarse el caso de Rumanía.

Se da la circunstancia, sin embargo, de algún ensayo determinado, como es el caso del LOKTEST, desarrollado en Dinamarca, en el que los resultados sirven de base para el control de recepción del hormigón, sustituyendo en muchas ocasiones al ensayo clásico de probetas moldeadas de control.

V. M. Malhotra, en su comunicación relativa al resumen global de los aspectos tratados en la convención de ACI, que se celebró en Ottawa (Canadá) en 1984 (16), ya señaló que, aunque se ha avanzado mucho en la normalización del uso de estos métodos, a través de organizaciones como ASTM, BSI, DIN, ISO, RILEM y ACI y puede afirmarse que hay acuerdo en el contenido de dichas normativas; existen, sin embargo, bastantes puntos de vista diferentes.

Considera Malhotra que se ha de desarrollar una mayor investigación sobre la aplicación de estos métodos en estructuras reales, con la consiguiente determinación de los niveles de precisión que se obtengan en cada caso para los parámetros investigados, antes de que puedan ser introducidos en las correspondientes normativas o códigos como criterios de Control de Calidad del hormigón.
(1) ACI-214-77 Reaffirmed (1983): "Recommended practice for evaluation of strength test results of concrete."

(2) ASTM-C-42-82: "Standard method of obtaining and testing drilled cores and vawed beams of concrete."

(3) BS-6.089 (1981): "Assessment of concrete strenth in existing structures", London.
(4) BS-1.881, part. 120. (1983): "Testing concrete. Method for determination of the compressive strength of concrete cores", London.

(5) BS 1881, part. c 201 (1986): "Testing concrete. Guide to the use of non-destructive methods of tests for hardened concrete".

(6) BS 1.881, part. 202 (1986): "Testing concrete. Recommendations for surface hardness testing by rebound hammer", London. 
(7) BS 1881, part. 203 (1986): "Testing concrete. Recommendations for measurement of velocity of ultrasonic pulses in concrete", London.

(8) BUNGEY, J. H. (1984): "The influence of reinforcement on ultrasonic pulse velocity testing. ACI. In situ NDT of concrete. Otawa.

(9) CAFFARENA, Jorge: "Influencia de las armaduras de pequeños diámetros, en la resistencia a compresión de testigos de hormigón."

(10) CHUNG, H. W.: "Effects of embebed steel bars upon ultrasonic testing of concrete", Magazine of Concrete Research, volumen 30, número 102.

(11) EH-91: "Instrucción para el proyecto y la ejecución de obras de hormigón en masa o armado", Comisión Permanente del Hormigón.

(12) IRANOR. UNE-83.302 (1984): "Extracción y conservación de probetas testigo del hormigón."

(13) IRANOR. UNE-83.307-86 (1986): "Ensayos de hormigón. Determinación del Indice de rebote."

(14) IRANOR. UNE-83.308-86 (1986): "Ensayos de hormigón. Determinación de la velocidad de propagación de los impulsos ultrasónicos."
(15) JONES, R., y FACAOARU, I. (1968): "Analysis to answers to a questionaire on the ultrasonic pulse technique", RILEM, París.

(16) MALHOTRA, V. M. (1984): "In situ non destructive testing of concrete. A global review", $A C I$. In situ NDT of concrete. Ottawa.

(17) MUNDAY, J. G. L., y DHIR, R. K. (1984): "Assessment of in situ concrete queality by core testing", $A C I$. In situ NDT of concrete, Ottawa.

(18) PETERSONS, N.: "Recommendations for estimation of quality of concrete in finished structures", Bulletin RILEm, número 24 .

(19) RIRELM. Recommendations NDTD-1 (1969): "Testing of concrete by the ultrasonic pulse method", Materiaux et Constructions, volumen 2, número 10

(20) RILEM. Recommendations NDT-3 (1983): "Testing concrete by hardness methods. 43-CND. Final recommendations", Materiaux et Constructions, volumen 16, número 95. .

(21) RILEM (1991): "Quality Control of concrete structures", Proceedings of the Second International RILEM/CEB Symposium. GHENT, junio.

\section{Publicación del Instituto Eduardo Torroja - CSIC}

\section{Número monográfico de INFORMES}

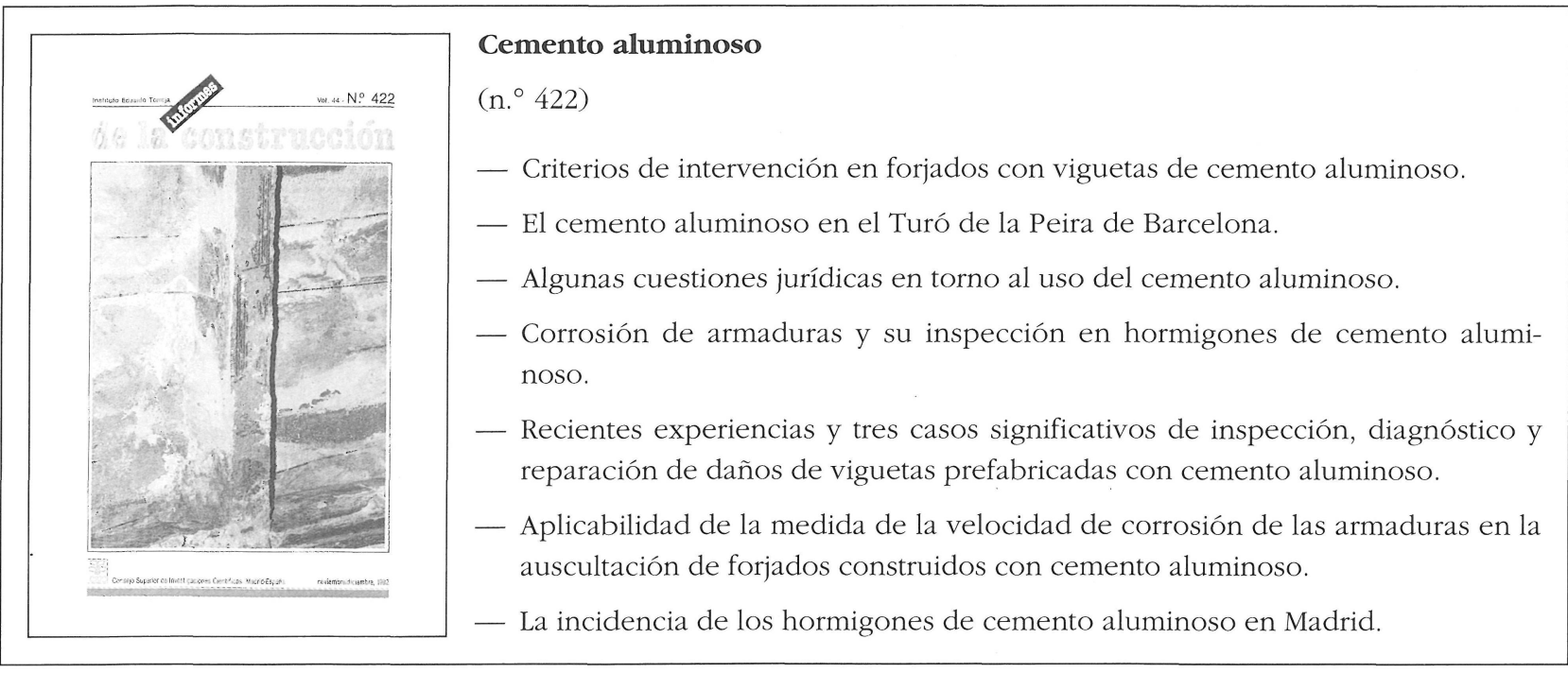

